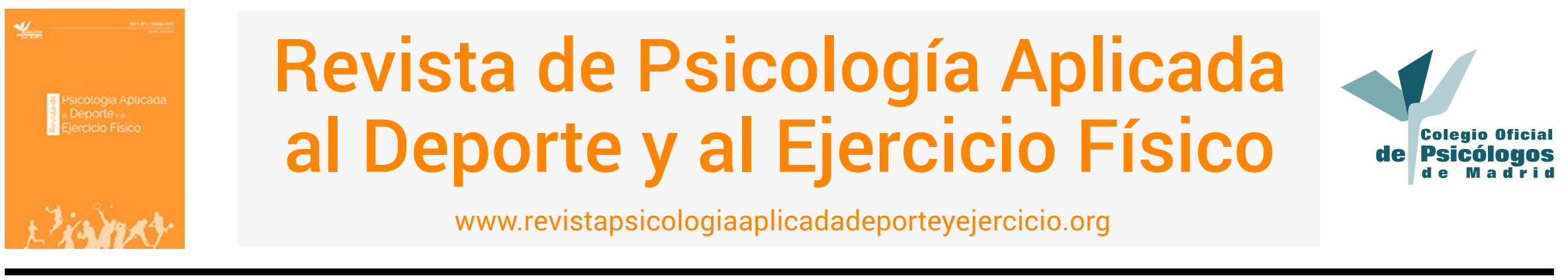

\title{
El Entrenamiento Psicológico-Integrado en Fútbol a través de Tareas de Entrenamiento
}

\author{
Juan Martín Tassi', Santiago Rivera Matiz ${ }^{2}$ y Miguel Morilla Cabezas ${ }^{3}$ \\ 'Universidad de Extremadura, España. ${ }^{2}$ Centro Excelentia, España. ${ }^{3}$ Universidad Pablo de Olavide, España
}

RESUMEN: A pesar de la relevancia que tiene el componente psicológico en el entrenamiento deportivo, en la actualidad su integración específica dentro de las sesiones de trabajo es todavía limitada y alejada de la realidad del juego. No obstante, en las tareas con balón este resulta indispensable para que el futbolista aprenda a comprender el juego, es decir, a tomar decisiones correctas (competencia táctica) y a ejecutarlas adecuadamente (competencia técnica). Este artículo tiene como objetivo ofrecer orientaciones sobre cómo integrar la preparación de las habilidades psicológicas con el resto de las capacidades y aspectos del juego (táctica, técnica, condicionales) mediante tareas de entrenamiento. Para ello se tienen en consideración las situaciones integradas demandadas por la competición, en donde se acentúan los contenidos del componente psicológico que especialmente interese mejorar por medio de la regulación de la carga psicológica; y dicho cometido se llevará a cabo mediante el diseño de tareas o ejercicios con balón.

PALABRAS CLAVES: Entrenamiento, método integrado, carga psicológica y fútbol.

\section{Integrated psychological training in football through training tasks}

ABSTRACT: Despite the relevance of the psychological component in sports training, its specific integration in work sessions is still limited and far from the reality of the game. However, in tasks with the ball it is essential for the player to learn to understand the game, that is, to make adequate decisions (tactical competence) and to execute them properly (technical competence). This article aims to offer guidance on how to integrate the preparation of psychological skills with all other skills and aspects of the game (tactical, technical, conditional) through training tasks. For this, the integrated situations demanded by the competition are taken into account, and the contents of the psychological component that are especially interesting to improve are accentuated through regulation of the psychological burden. This task will be carried out through the design of tasks or exercises with the ball.

KEYWORDS: Training, integrated method, psychological load and football.

\section{Treino integrado psicológico no futebol através de tarefas de treino}

RESUMO: Apesar da relevância da componente psicológica no treino desportivo, atualmente a sua integração específica nas sessões de trabalho ainda é limitada e distante da realidade do jogo. No entanto, em tarefas com a bola, é essencial que o jogador aprenda a entender o jogo, ou seja, a tomar decisões corretas (competência tática) e executá-las adequadamente (competência técnica). Este artigo tem como objetivo oferecer orientações sobre como integrar a preparação de competências psicológicas com as restantes competências e aspetos do jogo (táticos, técnicos, condicionais) através de tarefas de treino. Para tal, as situações integradas exigidas pela competição são levadas em conta, onde se destacam os conteúdos da componente psicológica que interesse especialmente melhorar através da regulação da carga psicológica; e esta tarefa será realizada através da conceção de tarefas ou exercícios com bola.

PALAVRAS-CHAVE: Treino, método integrado, carga psicológica e futebol.

Artículo recibido: 22/02/2018 | Artículo aceptado: 20/04/2018

Juan Martín Tassi, es preparador físico. Club Estudiantes de La Plata, Argentina; Santiago Rivera Matiz, es psicólogo. Director del Centro Excelentia; Miguel Morilla Cabezas, es psicólogo. Profesor Universidad Pablo de Olavide, España 
En el deporte actual la relevancia del componente psicológico en el entrenamiento deportivo es incuestionable. No obstante, su integración específica dentro de las sesiones de trabajo es todavía limitada y alejada de la realidad del juego (Morilla y Rivera, 2014); sin embargo, es allí, con la presencia del balón de fútbol, mediante tareas de trabajo, donde el jugador mejora las cualidades técnico-tácticas y donde debe entrenar el componente psicológico (Morilla, Rivera y Tassi, 2017). Este artículo tiene como objetivo ofrecer orientaciones sobre cómo integrar la preparación de las habilidades psicológicas con el resto de las capacidades y aspectos del juego (p. ej., táctica, técnica, condicionales) mediante tareas de entrenamiento, con mayor énfasis en las situaciones integradas demandadas por la competición, y acentuando los contenidos del componente psicológico que especialmente interese mejorar; y dicho cometido se llevará a cabo mediante el diseño de tareas o ejercicios con balón.

Para ello, es indispensable conocer aquellos factores que componen la esencia y lógica del deporte, donde el componente táctico, el modelo de juego y sus demandas son el primer paso para entrenar las variables que conforman el juego. A partir de su comprensión, es posible identificar las necesidades del jugador y los medios para enseñarle las habilidades que le permitan afrontar y resolver los distintos retos deportivos.

El fútbol como deporte cuenta con las siguientes características: 1) es sociomotriz, por lo que el jugador actúa en relación con otros jugadores y en presencia de incertidumbre por parte del medio, compañeros y oponentes (Parlebas, 1981); 2) se regula externamente, lo que implica tareas que requieren de resolución de problemas a partir de la situación de juego (Seirul·lo, 2003); 3) es situacional, donde predominan las habilidades motrices de tipo abierto con numerosas alternativas de solución (Seirul·lo, 2003); y 4) el espacio y tipo de participación, que en el fútbol es compartido por los jugadores de forma simultánea (Hernández-Moreno, 1994).

Dadas las anteriores características del deporte, así como las exigencias del fútbol actual según FIFA (2014) y UEFA (2016), tales como la (inteligencia, la versatilidad, la creatividad, o la fortaleza física, mental y emocional; surgen cinco características principales y específicas de fútbol (adaptado de Morilla y Rivera, 2014):

- Competitividad: componente intrínseco del deporte en el marco de los valores afines al juego limpio.

- Colaboración: necesaria para coordinar esfuerzos, trabajar en equipo, potenciar los recursos del sistema y, en definitiva, jugar mejor.

- Oposición: hay dos equipos que buscan marcar goles y evitar encajarlos.
- Intensidad: el juego se desarrolla con rapidez y en un espacio limitado.

- Incertidumbre: el resultado de las acciones es incierto y depende de distintos factores externos que difícilmente se pueden controlar, como las decisiones y el comportamiento de los compañeros de equipo y de los oponentes.

\section{Componentes estructurales de juego}

El fútbol está compuesto por distintas partes que forman una estructura compleja, las cuales han de comprenderse para poder adaptarlas a fin de diseñar las tareas de entrenamiento y acentuarlas en el componente psicológico:

- El espacio de juego: aspecto que orienta la actuación individual y colectiva en cada situación del juego, bien en el proceso ofensivo (p. ej., se busca ampliarlo) o defensivo (p. ej., se busca reducirlo), y su correcta utilización (Ardá y Casal, 2003).

- El reglamento en la competición y los entrenamientos: consiste en un sistema de reglas o normas que definen la lógica y el desarrollo del juego. Según Hernández Moreno (1994) este abarca dos dimensiones: 1) la formal (p. ej., características del espacio, número de jugadores, tiempo de juego, etc.) y la funcional (p. ej., forma de jugar, de utilizar el espacio, de sancionar, etc.); y 2) las estrategias utilizadas durante la semana en las tareas para favorecer el aprendizaje y desarrollo de conductas esperadas acorde a los objetivos de la sesión de entrenamiento.

- El tiempo: tiene gran importancia para analizar el desarrollo de la acción en el juego. Influye sobre el adelanto de la ejecución técnica (p. ej., acto motor), en la utilización del espacio (p. ej., a más espacio más tiempo para actuar), y en el número de acciones individuales o colectivas por unidades de tiempo (p. ej., ritmo de juego), según indican Ardá y Casal (2003). Gracias a estas implicaciones, se puede crear incertidumbre en el equipo rival jugando con la ocupación, anulación o creación de espacios, y abocándolo a actuar en el menor tiempo posible (Vegas, Romero y Pino, 2012).

- La técnica o modelos de ejecución: La técnica no tiene cabida en el juego sino se encuentra dentro de unos marcos tácticos y estratégicos adecuados; así también la táctica y la estrategia no conseguirán sus objetivos si la aplicación técnica no es la adecuada (Vegas et. al, 2012).

- La comunicación motriz y la relación de cooperación/oposición: en el fútbol se da una comunicación motora (p.ej., con señales, gestos o la transmisión del balón) entre los jugadores de un mismo equipo y los adversarios, a través de la cual se posibilita el desarrollo y ejecución de determinadas situaciones de juego (Vegas et. al, 2012). 
- La estrategia motriz: para jugar con éxito el futbolista debe conocer la realidad del juego y analizar en todo momento la situación con el fin de actuar de acuerdo a ella (Ardá y Casal, 2003).

\section{Componentes del rendimiento}

Los contenidos de la preparación del futbolista han de estar vinculados y globalizados para aplicarse a la práctica deportiva en entrenamientos y competiciones. De este modo se facilita la transferencia de los aprendizajes, abarcando los ámbitos que le acerquen a lo que se va encontrar en la situación real de partido (Morilla y Rivera, 2014). Entre estos, encontramos los siguientes:

- Componente condicional y coordinativo: incluye capacidades básicas o condicionales (p. ej., fuerza, velocidad, resistencia y flexibilidad) y complementarias o coordinativas ( $p$. ej., coordinación, agilidad, equilibrio, ritmo, etc.).

- Componente técnico: en el fútbol se requiere más de una técnica rápida para dar seguridad al dominio del balón con velocidad en la ejecución, para lo cual se ha de prestar atención a la situación de juego, ganar ventaja táctica y desarrollar un fútbol más rápido, imaginativo y creativo (Casáis y Conde, 2008). Las acciones técnicas son de carácter individual o colectivo, y ofensivas o defensivas.

- Componente táctico: para superar al equipo contrario es necesario anticiparse, sorprenderlo o engañarlo; por lo tanto, resulta fundamental saber cuándo, dónde y por qué realizar las acciones tácticas (Casáis, Domínguez y Lago, 2008), de acuerdo al modelo de juego o modelo táctico que sigue el entrenador. La táctica en el fútbol es individual o colectiva, y ofensiva o defensiva.

- Componente psicológico y socioafectivo: las habilidades psicológicas condicionan el juego, pues todo comportamiento deportivo requiere de la puesta en marcha de distintos procesos mentales: aprendizaje, percepción, atención, memoria, pensamiento, lenguaje o inteligencia; los cuales explican el comportamiento en las personas (Pérez, Gutiérrez, García y Gómez, 2005). En el futbolista y en su contexto específico, funcionan como un sistema de capacidades psicológicas (Morilla et al., 2017) que interactúan permitiéndole adaptarse y relacionarse en distintas situaciones: dentro (i.e. en entrenamientos y partidos) y fuera del terreno de juego (i.e. en los vestuarios, concentraciones, entrevistas, etc.).

Por otra parte, entre las distintas necesidades psicológicas y socioafectivas del futbolista, encontramos las siguientes: motivación, atención y concentración, control emocional, control de la activación, inteligencia, autoconfianza, cohesión grupal, liderazgo y comunicación (Beswick, 2012; Dosil, 2006; Morilla y Gómez, 2009; Morilla y Rivera, 2014; Olmedilla y Dominguez-Igual, 2016; Pulido et al., 2015; Roffé, 1999; Roffé y Rivera, 2014).

\section{Componentes funcionales}

Los componentes funcionales se constituyen de aquellos aspectos que definen las tareas que los futbolistas deben desempeñar en cada situación del juego, y de esta forma coordinar los esfuerzos individuales en beneficio del sistema y del equipo (Leo, García-Calvo, Sánchez, y De la Vega, 2011). Su funcionamiento está regido por los siguientes elementos (Casáis y Conde, 2008): las fases del juego, los principios de juego, los roles, acciones e intenciones tácticas.

\section{Conductas deportivas transversales}

Las conductas transversales específicas son aquellas que integran los componentes del rendimiento del fútbol ( $p$. ej., psicológico, condicional/coordinativo, técnico y táctico). Por ejemplo, estar atento a los movimientos de los compañeros y rivales dentro del campo (p. ej., psicológico), la velocidad en el desplazamiento para poder llegar al balón ( $p$. ej., condicional/coordinativo), su capacidad para controlarlo y pasarlo a un compañero (p. ej., técnico), y la interpretación y ejecución de los principios del juego (p. ej., táctico), confluyen en la representación de una situación de juego específica (Morilla et al., 2017). La posibilidad de poner en práctica cada uno de los componentes necesarios para su realización aumenta la probabilidad de alcanzar el objetivo en una tarea o situación de juego; y, por el contrario, no presentar alguna de estas conductas implica la ruptura en el encadenamiento de acciones y consecuente necesidad de reestructurar la interacción entre los jugadores.

Las conductas transversales específicas se presentan en situaciones reducidas/sectorizadas o globales de juego, se vinculan a la capacidad de combinar las variables y acciones que el fútbol demanda, y sus contenidos están asociados a los principios que establece el modelo de juego en concreto (Morilla et al., 2017).

Así, a partir de los aspectos de juego es posible determinar las aptitudes y actitudes que debe desarrollar el jugador para afrontar la competición. Por ejemplo, las conductas apropiadas de un equipo que quiere mantener la posesión del balón (p. ej., aspecto del juego específico en fase de ataque) requieren en cada componente del rendimiento que el jugador presente las capacidades indicadas en la Tabla 1. 
Tabla 1. Objetivos asociados (conductas transversales especificas)

\begin{tabular}{|c|c|c|c|c|}
\hline - Conservación & $\begin{array}{l}\text { - Utilización de } \\
\text { espacios libres, } \\
\text { desmarque y apoyos. }\end{array}$ & $\begin{array}{l}\text { - Pase y control } \\
\text { de balón. }\end{array}$ & $\begin{array}{l}\text { - Aceleración, } \\
\text { desaceleración y fuerza } \\
\text { específica. }\end{array}$ & $\begin{array}{l}\text { - Motivación para buscar línea de pase y } \\
\text { dar apoyos. } \\
\text { - Cambio de foco atencional amplio } \\
\text { externo y estrecho según la situación. } \\
\text { - Control de la activación que favorezca la } \\
\text { coordinación motriz-técnica. } \\
\text { - Comunicación, dentro de las consignas, a } \\
\text { partir del diálogo durante la tarea. }\end{array}$ \\
\hline
\end{tabular}

\section{Procesos psicológicos implicados en el comportamiento motor}

El fútbol actual es veloz, y la velocidad del juego está en la "cabeza" (UEFA, 2012), cuyo funcionamiento depende de la capacidad para interpretar y analizar las señales del juego (Mombaerts, 2000). En base al análisis que distintos autores han realizado sobre el mismo (Casáis et al., 2008; Mombaerts, 2000; Morilla et al., 2017; Vegas et al., 2012), entendemos que una de sus mayores dificultades radica en la cantidad de información externa que debe gestionar, en el poco tiempo del que dispone para hacerlo y en conseguir que su estado psicológico (p. ej., cognitivo y emocional) facilite ese análisis y su consecuente adaptación en búsqueda de la mejor solución motriz. En un entorno dinámico e incierto esta es una necesidad permanente.

Así, el resultado de dicho proceso se observa en el comportamiento motor, pero el mismo consiste en un proceso que cuenta con dos aspectos funcionales (Oña, Martínez, Moreno y Ruiz, 1999): 1) uno se encarga de recibir y organizar la información para preparar la respuesta (p. ej., cognitivo); y 2) otro de contenido energético que se refiere a la carga psico-biológica necesaria para llevarlo a cabo (p. ej., la activación).

En el futbolista, entendemos que el comportamiento motor se debe comprender de forma integral y seguir varias fases, las cuales se explican en la Tabla 2.

\section{Habilidades psicológicas que necesita entrenar el futbolista}

A partir del análisis del juego podemos definir las demandas psicológicas implicadas en el rendimiento del futbolista, las cuales se pueden aprender y por tanto incluir como contenidos de su preparación a fin de potenciar el proceso de aprendizaje y, consecuentemente, la eficacia táctica (Morilla et al., 2017). En la Tabla 3 se indica la habilidad concreta que se desprende de cada característica del fútbol.

Tabla 2. Fases del comportamiento motor del futbolista (En Morilla y Rivera, 2014, adaptado de Fradua, 1997; y Malho, 1969)

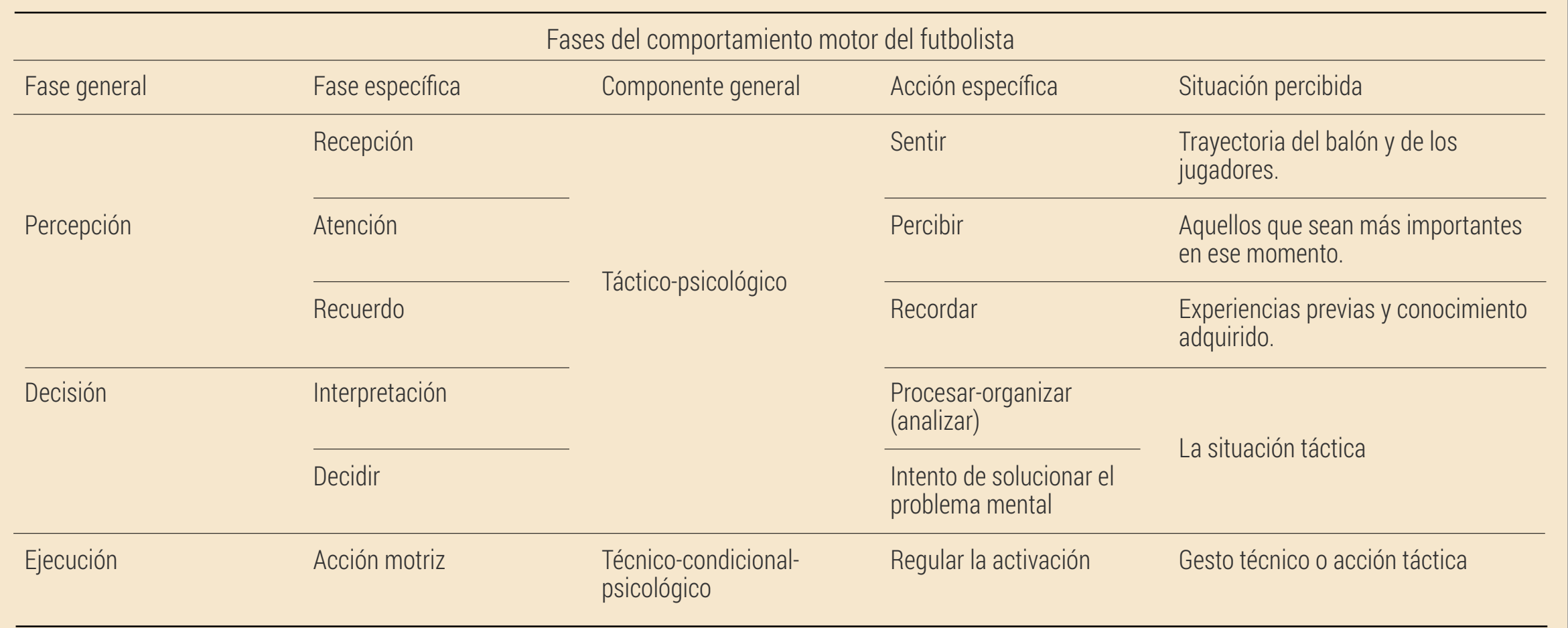


Tabla 3. Características del fútbol, necesidades psicológicas y habilidades a entrenar (adaptado de Morilla y Rivera, 2014)

\begin{tabular}{|c|c|c|}
\hline Características del fútbol & Necesidades psicológicas & Habilidades a entrenar (contenidos) \\
\hline 2. Colaboración & Cohesión grupal & $\begin{array}{l}\text { - Atención y concentración. } \\
\text { - Comunicación verbal, no verbal y motriz. } \\
\text { - Inteligencia emocional. } \\
\text { - Liderazgo. }\end{array}$ \\
\hline 3. Oposición & Anticipación & $\begin{array}{l}\text { - Activación-Intensidad deportiva. } \\
\text { - Contra comunicación motriz. } \\
\text { - Inteligencia (todas). }\end{array}$ \\
\hline 4. Incertidumbre & Adaptación & $\begin{array}{l}\text { - Atención y concentración. } \\
\text { - Inteligencia emocional. } \\
\text { - Activación. } \\
\text { - Autoconfianza. }\end{array}$ \\
\hline 5. Velocidad / Intensidad & Decidir rápido & $\begin{array}{l}\text { - Motivación. } \\
\text { - Percepción, toma de decisión y ejecución (o movimiento táctico) } \\
\text { - Activación. }\end{array}$ \\
\hline
\end{tabular}

\section{La carga psicológica en el futbol}

No cabe duda que el fútbol es un deporte complejo en lo psicológico: en el aspecto mental, requiere del rápido procesamiento cognitivo de muchos estímulos para tomar decisiones correctas en un ambiente dinámico; y en lo emocional, ese ambiente cambia constantemente y exige de una permanente adaptación a la incertidumbre (p. ej., miedo a no tener el control sobre el resultado) y sus consecuentes reacciones. Así, cada situación de juego pone a prueba la habilidad para sincronizar los recursos cognitivos y emocionales, poniéndolos al servicio del comportamiento técnico-táctico (Morilla et al., 2017).

Por lo tanto, la dificultad psicológica que enfrenta el futbolista en cada situación de juego viene determinada por su carga psicológica. Habitualmente se hace referencia a la misma como carga mental, pero Morilla et al. (2017) les resulta más apropiado hablar de la primera. El motivo es el siguiente: a partir de una comprensión de las personas desde una perspectiva integral u holística, no sólo se trabaja con su dimensión mental, la cual hace referencia al ámbito cognitivo del ser humano. Cárdenas, Conde-González y Perales (2015) sugieren que cuando se hable de carga mental también se puede hablar de carga emocional. Por lo tanto, entendemos que lo psicológico está compuesto por: 1) lo cognitivo (p. ej., prestar atención, procesar información o tomar decisiones); y 2) lo emocional (p. ej., gestionar el miedo, la ira o la alegría).

De esta forma Morilla et al. (2017) adaptan la definición de carga mental de DiDoménico y Nussabaum (2008) y formulan una más integradora la cual entiende que la carga psicológica se define como el coste de recursos cognitivos y emocionales que le supone al deportista para conseguir un nivel de rendimiento determinado en una tarea con demandas específicas internas o externas; y en concreto para un futbolista, define la cantidad de recursos psicológicos que debe utilizar para adaptarse y resolver una situación de juego en un entorno determinado. La misma contempla los siguientes conceptos clave:

- Coste de recursos: consumo o gasto de recursos psicológicos de un jugador cuya utilización implica un esfuerzo (p. ej., mantener la concentración o tolerar la frustración). 
- Recursos psicológicos: capacidades y medios cognitivos y emocionales que sirven al deportista para jugar al fútbol, que son limitados y se agotan como consecuencia del esfuerzo prolongado (p. ej., motivación, atención y concentración, etc.).

- Demanda interna de la tarea: recursos psicológicos necesarios para desempeñarse en una tarea o situación de juego (p. ej., mantener la concentración en una actividad que no se perciba motivante o cuando se sienta frustración).

- Demanda externa de la tarea: recursos necesarios para adaptarse a las características del entorno de una tarea y encontrar las oportunidades que este ofrece (p. ej., presión del público o campo en mal estado).

- Adaptación del futbolista: proceso por medio del cual el jugador ajusta o modifica sus recursos psicológicos según lo requiera la tarea (p. ej., deportista que mejora su orientación corporal y para jugar mejor en espacios reducidos, o que compite en un terreno de juego en malas condiciones)

Una tarea más compleja supone una mayor carga psicológica, y una más simple, una menor, y en este sentido al hablar de complejidad en la metodología del entrenamiento psicológico-integrado, cobra importancia el concepto de incertidumbre (Morilla et al., 2017). Esta es una característica intrínseca del fútbol y de los deportes abiertos o condicionados por la situación. Por todo esto los jugadores toman decisiones en función de lo que hacen sus compañeros y oponentes, y están a expensas del comportamiento de otras personas. El cambio permanente y la inestabilidad es propio de la dinámica generada cuando los deportistas interactúan en un sistema (p. ej., equipo), con otros sistemas (p. ej., equipo oponente) y con el resto de los componentes de un sistema mayor (p. ej., partido de fútbol). Todos ellos crean un entorno incierto y complejo.

Es entonces cuando se hace necesario entrenar para potenciar el aprendizaje de habilidades psicológicas y el desarrollo de los recursos motores, cognitivos y emocionales; todos los cuales ayudan a solucionar las situaciones-problema de juego y consecuentemente, a reducir la incertidumbre facilitando la anticipación. De acuerdo con Cárdenas et al. (2015), cuanta más incertidumbre contenga una tarea, mayor será la cantidad de recursos que requiera, mayor carga de memoria de trabajo, más control atencional, más implicación de los procesos inhibitorios necesitará, y más demandas emocionales impondrá; es decir, mayor será el peso de la carga psicológica y mayor será el esfuerzo.

De esta forma, dicho peso debe gestionarse siguiendo una lógica que favorezca el proceso de aprendizaje y la ca- pacidad de adaptación del futbolista (Morilla et al., 2017). Para ello, el grado de incertidumbre que genera una tarea debe estar en un rango que supere por poco la capacidad del individuo para afrontarla (Cárdenas, Conde-González y Perales, 2017). De lo contrario, se corre el riesgo de que el proceso de adaptación pueda fracasar y resultar en síndromes como el burnout o el sobreentrenamiento (Cárdenas et al., 2015). Dicho aspecto debe tener en cuenta para el diseño de tareas y la progresión metodológica de su complejidad.

\section{La carga cognitiva en el fútbol}

Los procesos y recursos cognitivos que demanda el fútbol vienen dados por la participación simultánea de compañeros y adversarios en el mismo terreno de juego, lo cual hace, como se ha mencionado antes, que las maniobras de los jugadores en este espacio sociomotor posean gran complejidad (Ardá y Casal, 2003). Ante esta situación abierta y de adaptación permanente, para el jugador resulta indispensable su capacidad de análisis y decisión frente a la variedad de soluciones, y por supuesto disponer de los recursos técnicos para una correcta ejecución (Mombaerts, 2000).

El proceso mediante el cual se interpreta tácticamente una situación de juego (p. ej., prestar atención o tomar decisiones) requiere de esfuerzo, y ese esfuerzo viene dado por la carga cognitiva presente en la tarea. Esta exige una determinada cantidad de recursos para poderse resolver, todos los cuáles afecta dicho proceso de decisión. Por consiguiente, soportar la carga permite que la acción individual siga la lógica de un modelo de juego y ayude a los futbolistas a coordinar sus esfuerzos en la consecución de un objetivo común (Morilla et al., 2017).

En este sentido la dificultad radica en la cantidad de información y del nivel de procesamiento no automático que demanda esta información sobre el sistema cognitivo (Cárdenas et al., 2017). Cuando la situación se desconoce, la búsqueda de una respuesta se vuelve más lenta porque se percibe con mayor incertidumbre, esto aumenta la presión y puede bloquear el sistema de representación mental que contiene la información motora, paralizando el enlace perceptivo-cognitivo-motor y volviéndolo menos funcional (Tenenbaum y Gershgoren, 2014). Por esto el deportista inexperto necesita de un mayor esfuerzo mental para soportar la misma carga cognitiva que uno experto para adaptarse a la incertidumbre propia del fútbol. 


\section{La carga emocional en el fútbol}

El procesamiento de información en cualquier situación de juego está condicionado por las emociones que experimenta el jugador, pues estas constituyen influencias que afectan la tarea provocando interferencias, o bien facilitándola o modulando los recursos disponibles (Cárdenas et al., 2015).

La competición es intrínseca al deporte y puede resultar muy estresante cuando el riesgo de fracasar es percibido como algo que resta beneficios personales, sociales, o económicos. Es por esto que en las etapas formativas la cooperación resulta más educativa que la mera competición donde el niño carece de recursos para afrontar tanta presión. Por otra parte, cuando el deporte es el trabajo de un adulto, para los jugadores profesionales cada partido está sobrecargado de estrés emocional y social (Tenenbaum y Gershgoren, 2014), el cual forma parte del juego y ha de aprender a gestionarse.

El peso que debe cargar emocionalmente un jugador es lo mismo que la carga emocional. En la iniciación esta mochila debe ser muy liviana, y progresivamente, la misma, se ha de volver más pesada, y por tanto las tareas exigirán mayores recursos (p. ej., inteligencia emocional); y esas condicionan la capacidad de aprendizaje y el desempeño deportivo (Morilla et al., 2017).

Cuando aumenta la complejidad de la tarea, aumenta la incertidumbre; cuando la incertidumbre se incrementa, la tarea exigirá más demandas emocionales (Cárdenas et al., 2015). Como vemos, el entrenamiento psicológico-integrado requiere, además de la estimulación cognitiva (p. ej., directri- ces tácticas) del futbolista, más demanda del componente volitivo y emocional. Por ejemplo, una situación con demasiada dificultad puede resultar muy estresante y terminar desmotivando al jugador al percibir que un eventual fracaso amenaza excesivamente su autoestima; o si la encuentra muy simple y fácil de realizar ésta será poco tentadora como para esforzarse por realizarla. En relación a la atención y concentración, estas también se ven afectadas por la carga emocional, estrechando el foco atencional a uno centrado en la situación amenazante, y dificultando la alternancia a uno amplio que permita encontrar otras soluciones dentro de la situación juego (Morilla et al., 2017).

La incertidumbre aumenta el nivel de estrés y puede disminuir la autoconfianza, pues decrece la certeza del deportista de contar con las capacidades para llevar a cabo una tarea. Por lo tanto, es responsabilidad del entrenador crear un ambiente de práctica que busque estados emocionales favorecedores del aprendizaje y rendimiento, es decir, aquellos que se perciban como positivos; y en su justa medida, el nivel de estrés manipulando las tareas y el tipo de feedback. En relación a este último, las consecuencias de la propia conducta durante el entrenamiento pueden utilizarse como factor generador de emociones positivas y negativas (Cárdenas et al., 2015). Por otro lado y en esa línea, una situación previa de competición (p.ej., acondicionamiento pre-competitivo) podrá favorecer este tipo de ambientes a través de situaciones de superioridad numérica, en donde la fluidez y dinámica de la tarea favorezcan, por ejemplo, los estados de autoconfianza (y autoeficacia colectiva) antes de los partidos (Morilla et al., 2017). Ver Figura 1.

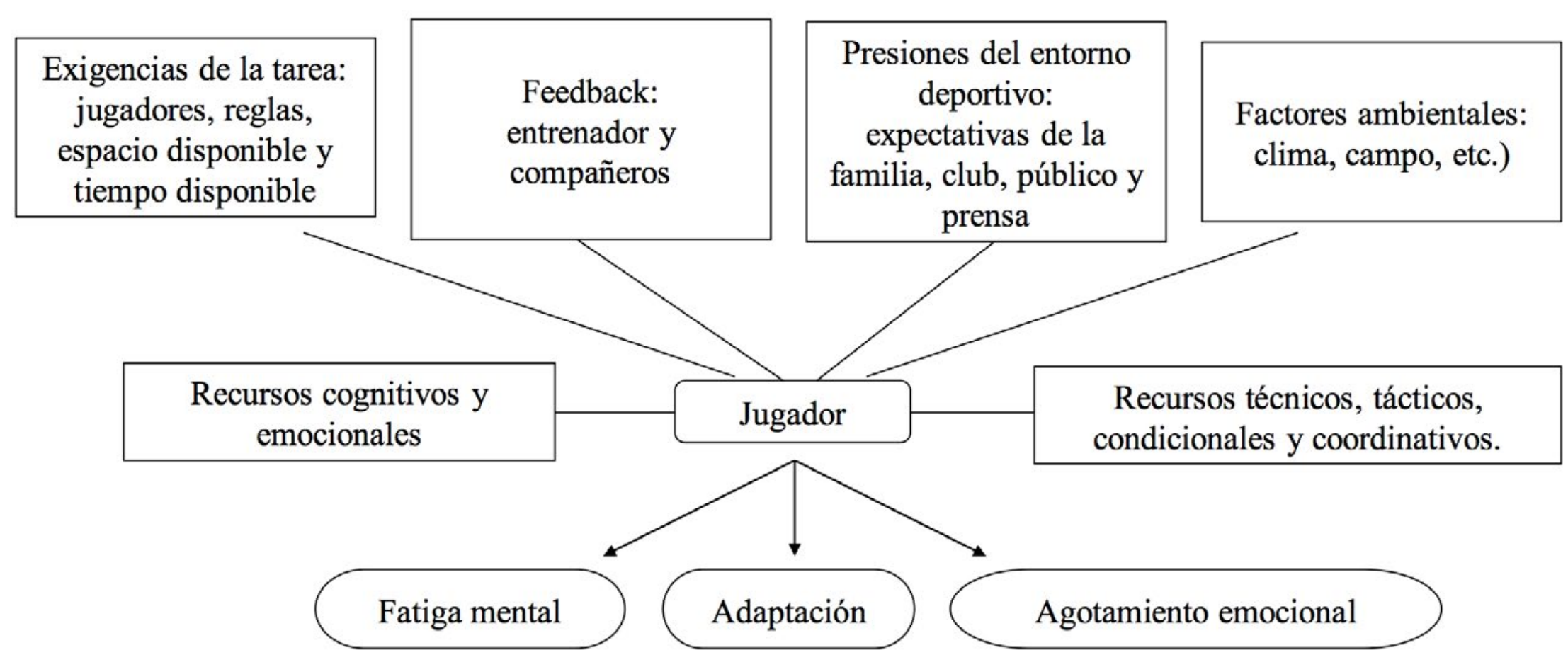

Figura 1. Factores que configuran la carga psicológica (En Morilla et al., 2017; adaptado de De Arquer, 1999; y Cárdenas et al., 2015). 
Como se observa, la carga psicológica está configurada por aspectos que se han de gestionar si también se quiere prevenir la fatiga mental y sus consecuencias en los jugadores. Esta se deriva de la actividad de naturaleza táctica, propia de los deportes de interacción motriz como el fútbol, en los que el deportista invierte un esfuerzo cognitivo para la toma de decisiones y otro conducente a la autorregulación emocional; lo cual pueden disminuir o agotar los recursos de un individuo para hacer frente a tareas que impliquen un esfuerzo de autocontrol, ya sea de la atención, cognitivo o emocional (Cárdenas et al., 2017). Por lo tanto, el entrenador ha de regular la carga psicológica para establecer las condiciones que favorezcan el aprendizaje y adaptación del futbolista a las tareas de entrenamiento y así prevenir que se fatigue mentalmente.

\section{Metodología del entrenamiento psicológico- integrado en el futbol}

Este artículo presenta una introducción al aprendizaje de las habilidades psicológicas integradas en la práctica del fútbol por medio de las tareas de entrenamiento, y cuya metodología debe adecuarse a la forma en que se entrena en este deporte.

\section{Metodología del entrenamiento en el futbol}

Los dos métodos predominantes en la enseñanza y entrenamiento del fútbol han sido el método analítico y el integrado-global (Pascual, Alzamora, Martínez y Pérez, 2015), los cuáles cuentan con las siguientes características:

- El método analítico: representado a partir de acciones de juego aisladas, de manera que sólo tiene en cuenta alguna de los elementos que intervienen en la competición, como lo físico o lo técnico. Por ejemplo, un grupo reducido de jugadores que desarrolla un circuito de pases con balón (p.e. control y pase corto) donde no existe incertidumbre y donde se desarrolla el gesto técnico a partir de la repetición del ejercicio y las correcciones del entrenador.

- Método integrado-global: se basa en la práctica deportiva donde las habilidades técnico-tácticas, condicionales y psicológicas se expresan de forma relacionada pero diferenciadas en su observación y análisis. Esta metodología contempla una integración y combinación entre diferentes tipos de carga, pero el común denominador es siempre la técnica y la táctica. Sans y Frattarola (2009) indican que este método facilita el desarrollo de las restantes cualidades que intervienen en el rendimiento, trabajándolos con- junta y genéricamente. Por ejemplo, en una situación de $3 \times 3$ donde el objetivo técnico-táctico ofensivo busca la mejora del desmarque y la progresión mediante el pase corto y el control de balón, y el objetivo defensivo se orienta a la presión al jugador con balón para intentar robarlo. Otro ejemplo puede ser una situación de 8x8 con los jugadores en posiciones habituales de juego, donde los objetivos se relacionan con las asociaciones específicas en campo (entre mediocampistas y delanteros contra defensores y mediocampistas) a partir de los principios o el modelo de juego que plantea el entrenador.

Por su parte, los modelos de planificación en el futbol se basan generalmente en una metodología integrada-global, cuyos exponentes, según Acero, Seirul·lo, Lago y Lalin (2013) son: 1) el microciclo estructurado, en el que la propuesta teórica consiste en el desarrollo de situaciones similares a la competición como medio para la optimización de las diferentes estructuras asociadas al juego (Seirul.lo, 2003); y 2) la periodización táctica, la cual propone una planificación sistémica que busca desarrollar el modelo de juego de un equipo apoyándose en principios, es decir, en comportamientos generales del juego, organizados de forma jerarquizada y congruente entre sí, que el entrenador ha creado y definido (Arjol, 2012). Tanto el microciclo estructurado como la periodización táctica se fundamentan en una planificación semanal (p. ej., microciclo), donde los objetivos se relacionan con una planificación anual, por lo general del futbol de formación; o entre competiciones, en el alto rendimiento (Arjol, 2012).

\section{Aplicaciones prácticas}

A partir de las propuestas metodológicas tradicionales de entrenamiento en fútbol, se propone un abordaje un poco más integrador para aplicar el entrenamiento psicológico-integrado. El mismo se puede adaptar a la reproducción en los entrenamientos de situaciones vividas en la competición, teniendo en cuenta los condicionantes psicológicos especialmente en el comportamiento táctico; a partir de la búsqueda de objetivos asociados al modelo de juego, manipulando los parámetros estructurales y psicológicos.

A continuación se describen los dos métodos propuestos para utilizar en el diseño de tareas y ejercicios psicológicos integrados (Morilla, et al., 2017):

- Analítico integrado: es analítico por el número de repeticiones y el planteo de un objetivo por componente, e integrado por el desarrollo conjunto de las capacidades asociadas al fútbol, mediante situaciones abiertas y reducidas o secto-

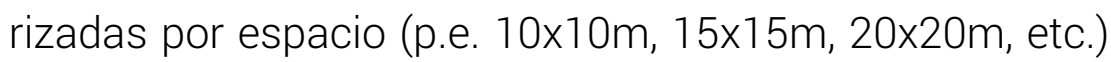


y número de jugadores (p.e. $2 \times 2,3 \times 3+2,3 \times 1,1 \times 2$, etc.). Por ende, nos encontraremos ante situaciones abiertas con objetivos específicos que se interrelacionan, con alta participación individual o colectiva.

- Integrado global: integrado por el trabajo conjunto de las capacidades asociadas al fútbol, y global por el desarrollo de situaciones abiertas y amplias (p.e. espacio con las dimensiones que requiere una tarea para dos líneas de juego contiguas, defensiva y mediocampo) y número de jugadores similares a la competición (p.e. 6×6+4), con menor cantidad de repeticiones, participación y número de interacciones entre jugadores, pero mayor relevancia del trabajo colectivo.

\section{Orientaciones pedagógicas para el diseño de ejercicios integrados acentuados}

A partir de la revisión de Morilla y Rivera (2014) y de las propuestas de varios autores (Cásais, Dominguez y Lago, 2008; Ardá y Casal, 2003; Fradua, 1997; Mombaerts, 2000; Morilla y Pérez, 2004), se recomienda tener en consideración los siguientes principios pedagógicos en el diseño de ejercicios y tareas para el entrenamiento psicológico dentro de la metodología integrada acentuada. Ver Tabla 4.
Tareas y contextos de juego: la progresión metodológica desde las situaciones analíticas hasta la competición

Todo diseño de tarea contemplará los condicionantes propios (p. ej., estilo de juego del equipo, fase de juego, metodología de trabajo, etc.), y determinarán la idoneidad o no de su perfil. Cualquier tarea requiere tanto la contextualización específica (p. ej., nivel de los jugadores, carga de trabajo, circunstancias ambientales, etc.) como la adecuación al momento en que la encuadremos ( $p$. ej., momento de la sesión de trabajo, momento de la temporada, etc.). Los siguientes son ejemplos de tareas en función de dichos aspectos (Morilla, et al., 2017).

Tareas analíticas generales. Las tareas analíticas buscan mejorar alguno de los elementos necesarios en el juego y suelen llevarse a cabo al inicio de la sesión (p.ej., pase, velocidad de reacción, atención en ejercicios con disonancia cognitiva, apoyo social, etc.). Por lo tanto, estos ejercicios buscan poner en marcha los procesos psicológicos que facilitarán la adaptación a las tareas, especialmente el aumento del nivel de activación necesario para orientar la atención hacia los objetivos de las tareas (ver Apéndice A).

Tareas analíticas integradas. Estas tareas permiten entrenar los cuatro componentes del rendimiento con un ma-

\section{Tabla 4. Orientaciones pedagógicas para el diseño de ejercicios integrados acentuados}

\section{Orientaciones pedagógicas para el diseño de ejercicios integrados acentuados}

Establecer objetivos cuantitativos y cualitativos, adaptados a las competencias individuales y colectivas en la tarea y competición.

Modificar la utilización y diseño de los elementos en relación a los cuatro aspectos o componentes del juego (i.e. táctico, técnico, condicional-coordinativo y psicológico-socioafectivo).

Tomar como premisa en las tareas o ejercicios la introducción de los elementos de juego (i.e. balón, compañeros, oponente, espacio y reglas), como condicionantes de las conductas de los jugadores en campo.

Acentuar los parámetros psicológicos (i.e. motivación, atención y concentración, activación, autoconfianza y cohesión grupal) con el fin de que estos favorezcan la comprensión del juego y la consecuente participación individual al servicio del colectivo; de acuerdo a la etapa de formación y al nivel de los jugadores.

Progresar de ejercicios analíticos integrados hacia integrados globales o viceversa según las necesidades individuales o colectivas del equipo (i.e. principio de reversibilidad).

Alternar situaciones de juego (p.e. espacios reducidos y amplios, número de jugadores, reglas, etc.) para que el jugador se vea en la necesidad de adaptarse a esfuerzos físicos cortos e intensos, al cambio de ritmo, a adecuar su mecanismo perceptivo y a cambiar de un foco atencional a otro, a ajustar su nivel de activación, a comunicarse permanentemente, etc. (i.e. modificar la carga psicológica).

Valorar el desarrollo de conductas asociadas a los aspectos tácticos (p.e. decisión correcta), condicionales (i.e. esfuerzo físico) y psicológicos (p.e. compromiso y perseverancia) por encima, en algunos casos, de los meros aspectos técnicos (p.e. acierto en la ejecución).

En etapas de formación el desarrollo de los aspectos técnicos se favorece en situaciones reducidas mientras que los aspectos tácticos y colectivos son favorecidos a través de situaciones globales.

Planificar dentro de una misma sesión ambos tipo de tareas (i.e. analíticas integradas e integradas globales). 
yor énfasis, según hemos explicado, en las situaciones con un número de jugadores reducido, así como trabajar las conductas individuales con interacciones simples, como rondos de 4 jugadores con dos defendiendo.

Dado que ofrecen la posibilidad de repetir o incidir sobre una cantidad reducida de contenidos, suponen un escalón en la progresión para mejorar una conducta transversal asociada a una situación específica de juego. Esto se lleva a cabo estableciendo dinámicas lo más cercanas posibles a los patrones psicomotrices y sociomotrices que ocurren en espacios reducidos (ver Apéndice B).

Tareas integradas globales. Según hemos visto, en estas tareas se incide sobre las conductas individuales dentro de un contexto colectivo más complejo, dada la ampliación del espacio y número de jugadores; donde se incorporan en una sola dos o más situaciones sectorizadas o reducidas, encadenando en el mismo ejercicio varias fases (p.ej., grupos de $1 \times 1+1$ se unen para formar un $2 \times 2+2,3 \times 3+3,5 \times 5+3$, etc.).

La progresión hacia situaciones de juego globales, viene dada por la aproximación de los componentes estructurales del juego tal como se presentan en la competición, y en este caso, con mayor atención en cómo los parámetros psicológicos y su consecuente carga mental y emocional condicionan el comportamiento motor (ver Apéndice C).

Competición. Las conductas transversales específicas se deberán transferir paulatinamente a las competiciones como consecuencia del proceso de enseñanza-aprendizaje (Morilla, et al., 2017); y las situaciones de competición deben tomar en cuenta la búsqueda de objetivos planteados en la continuidad del trabajo junto a los factores que interactúan en cada momento de la temporada (i.e. nivel del equipo, momento de los jugadores, condiciones de entrenamiento, etc.).

\section{Gestión de parámetros que regulen la carga cognitiva y emocional}

La metodología expuesta sugiere que las tareas de entrenamiento se acerquen a situaciones de juego similares a la competición, y para ello han de incluir los aspectos estructu-

Tabla 5. Nivel de carga psicológica y su relación con los elementos del juego

\begin{tabular}{|c|c|c|c|}
\hline $\begin{array}{l}\text { Componentes } \\
\text { carga psicológica }\end{array}$ & Baja complejidad & Media complejidad & Alta complejidad \\
\hline Espacio de juego & - Amplio. & - Habitual de juego. & - Reducido. \\
\hline Reglas & $\begin{array}{l}\text { - Mínimas modificadas. } \\
\text { - Generan poca incertidumbre. } \\
\text { - Perjudican poco. }\end{array}$ & $\begin{array}{l}\text { - Modificadas. } \\
\text { - Generan incertidumbre variable. } \\
\text { - En ocasiones perjudican. }\end{array}$ & $\begin{array}{l}\text { - Modificadas casualmente antes y } \\
\text { durante la tarea, o sin previo aviso. } \\
\text { - Generan mucha incertidumbre. } \\
\text { - Perjudican. }\end{array}$ \\
\hline Tiempo de resolución & $\begin{array}{l}\text { - Amplio que facilita la habituación. } \\
\text { - Menor intensidad. }\end{array}$ & - Habitual. & $\begin{array}{l}\text { - Reducido que previene la } \\
\text { habituación. } \\
\text { - Mayor intensidad. }\end{array}$ \\
\hline Jugadores & $\begin{array}{l}\text { - Menor densidad y oposición. } \\
\text { - Superioridad numérica. }\end{array}$ & $\begin{array}{l}\text { - Habitual de juego. } \\
\text { - Igualdad numérica. }\end{array}$ & $\begin{array}{l}\text { - Mayor densidad y oposición. } \\
\text { - Inferioridad numérica. }\end{array}$ \\
\hline Nivel de fatiga física & - Bajo. & - Moderado. & - Alto. \\
\hline Fases de juego: ataque y defensa & $\begin{array}{l}\text { - Mayor cooperación y menor } \\
\text { competición. }\end{array}$ & $\begin{array}{l}\text { - Comunicación motriz. } \\
\text { - Cooperación y competición. } \\
\text { - Oposición reducida. }\end{array}$ & $\begin{array}{l}\text { - Comunicación motriz. } \\
\text { - Cooperación y oposición. }\end{array}$ \\
\hline Tipo de tarea & $\begin{array}{l}\text { - Analítica integrada- integrada } \\
\text { global. }\end{array}$ & $\begin{array}{l}\text { - Analítica integrada - integrada } \\
\text { global. }\end{array}$ & $\begin{array}{l}\text { - Analítica integrada - integrada } \\
\text { global } \\
\text { - Competición. }\end{array}$ \\
\hline
\end{tabular}


rales propios del fútbol (p. ej., jugadores, espacio, balón, reglas, etc.) para acentuar o incidir en el trabajo sobre aquellos aspectos del juego que más interese mejorar en cada sesión.

El artículo se enfoca en la acentuación del componente psicológico (p. ej., motivación, atención y concentración, control de la activación, autoconfianza, y cohesión grupal), cuya dificultad está condicionada por la gestión de la carga cognitiva y emocional. En la Tabla 5 se observa cómo se comportan los elementos estructurales del juego en relación a la complejidad del componente psicológico (Morilla, et al., 2017).

A partir de los elementos o componentes del juego y la utilización de estrategias que favorezcan la variabilidad de situaciones psicológicas especificas, será posible modificar los niveles de incertidumbre. Esto facilita la activación y niveles de concentración, atención y motivación necesarios para las tareas y competición variando las siguientes condiciones (Morilla et al., 2017): 1) la ejecución de los encadenamientos; 2) el inicio (p. ej., forma de comenzar, situación de jugadores); 3) la oposición (p. ej., igualdad, superioridad numérica ofensiva o defensiva); y 4) la velocidad de la ejecución (p. ej., limitación de toques o intensidad).

Las características principales de las tareas analíticas integradas respecto a los elementos o componentes estructurales del juego se asocian a los siguientes aspectos:

- Secuencias cortas de alta intensidad respecto a la circulación de balón y participación individual sobre la tarea.

- Mayor cantidad de ejecuciones en cortos periodos de tiempo.

- Conocimiento y utilización de los espacios como recursos de juego (p.e. desmarques, juego asociado, presión sobre el balón, etc.)

- Alternancia de funciones de forma continua (p.e. posesión o no del balón, comodines) favoreciendo las transiciones rápidas).

- Número reducido de reglas aunque modificadas en algunos casos para favorecer las conductas deseadas.

- El balón como condicionante de los comportamientos de los jugadores con/sin balón en relación a otros elementos estructurales (p. ej., cantidad de jugadores en posesión por espacio, etc.).

Por su parte, las características principales de las tareas integradas globales respecto a los elementos/componentes estructurales del juego serían las siguientes:

- Dimensiones y espacios de juego similares a la competición.

- Interacciones de juego relacionadas con la competición.

- Encadenamiento de acciones colectivas (p. ej., conservación, progresión y finalización; ataque/defensa/ataque).

- Roles a partir de la posesión o no del balón y de acción directa o periférica.

- Facilitar el asentamiento de las conductas colectivas a partir de la superioridad numérica en la conservación y utilización del balón.

- Como se ha expuesto, las capacidades psicológicas serán parte de la planificación de las tareas, pero a su vez se utilizarán como componentes estructurales y estratégicos que permitan regular la carga psicológica (Cárdenas et al., 2015) en las sesiones de entrenamiento y durante un período determinado de la temporada (micro, macro o meso ciclo).

\section{Discusión}

El artículo aborda los aspectos fundamentales a tener en cuenta en el diseño de tareas de entrenamiento donde los contenidos psicológicos se pueden integrar con los demás componentes del rendimiento, así como acentuarlos según lo requiera el entrenador. Se considera que la metodología propuesta resulta apropiada para acercar la preparación de los jugadores a las demandas reales del fútbol, en cuanto optimiza la transferencia del aprendizaje a la competición; proceso que favorece la motivación al trabajo en el deportista al encontrar las tareas más estimulantes.

De igual manera, dicha propuesta de entrenamiento psicológico-integrado no sólo ofrece recursos metodológicos a los entrenadores, sino también a: 1) los psicólogos en cuanto les ayuda a comprender mejor el deporte y "hablar el mismo idioma" que los demás técnicos, y con ello poder asesorarles en el trabajo de campo; y 2) los preparadores físicos, a quienes ofrece recursos similares que a los entrenadores pero en las parcelas como el trabajo condicional, coordinativo y activador con presencia del balón.

\section{Agradecimientos}

El diseño gráfico de las tareas corresponde a la captura de pantalla de su versión en vídeo con el software 3D Futbol Táctico Coach, el cual ha sido cedido a los autores por Fútbol Táctico Group para su realización.

\section{Referencias}

Acero, R., Seirul|lo., F., Lago. C. y Lalin, C. (2013). Causas Objetivas de Planificación en DSEQ (II): La Microestructura (Microciclos). Revista de Entrenamiento Deportivo, 27(1), 1-19.

Ardá, T. y Casal, C. (2003). Metodología de la enseñanza en el fútbol. Barcelona, España: Editorial Paidotribo.

Arjol, J. L. (2012). La planificación del entrenamiento en futbol: análisis comparado del enfoque estructurado y la periodización táctica. Revista Científica Acción motriz, 8, 27-37. 
Beswick, B. (2012). Focused for soccer (2nd Ed.). Champaign, IL: Human Kinetics.

Cárdenas, D., Conde-González, J., y Perales, J. C. (2015). El papel de la carga mental en la planificación del entrenamiento deportivo. Revista de Psicología del Deporte, 24, 91-100.

Cárdenas D., Conde-González J. y Perales J. C. (2017). La fatiga como estado motivacional subjetivo. Revista Andaluza de Medicina del Deporte. 10(1), 31-41. https:/doi.org/10.1016/j. ramd.2016.04.001

Casáis, L y Conde, L. (2008). Fútbol base: el entrenamiento en categorías de formación. Pontevedra, España, Editorial: MC Sports.

Casáis, L., Domínguez, E. y Lago, C. (2008). Fútbol base: el entrenamiento en categorías de formación (Vol. I). Pontevedra, España: MC Sports.

De Arquer, M. I. (1999). Carga mental de trabajo: factores. Recuperado de: http://www. insht.es/InshtWeb/Contenidos/Documentacion/FichasTecnicas/NTP/Ficheros/501a600/ntp_534.pdf

Dosil, J. (2006). Psychological interventions with football (soccer) teams. En J. Dosil (Ed.), The Sport Psychologist's Handbook: A Guide for Sport-Specific Performance Enhancement, (pp. 139158). Chichester, Reino Unido: Jhon Wiley y Sons Ltd. https:/ doi.org/10.1002/9780470713174

Fradua, L. (1997). La visión de juego en el futbolista. Barcelona, España: Editorial Paidotribo.

FIFA (2014). 2014 FIFA World Cup Brasil Technical Report and Statistics. Recuperado de: http://resources.fifa.com/mm/document/footballdevelopment/technicalsupport/02/42/15/40/ 2014fwc_tsg_report_15082014web_neutral.pdf

Hernández Moreno, J. (1994). Fundamentos del deporte: Análisis de las estructuras de juego deportivo. Barcelona, España: INDE.

Leo, F., García-Calvo, T., Sánchez, P. y De la Vega, R. (2011). Relación entre la cohesión de equipo, la eficacia percibida y el rendimiento en equipos masculinos de jóvenes futbolistas. Revista de Iberoamericana de Psicología del Ejercicio y el Deporte, 6(1), 47-62.

Malho. (1969). La acción táctica en el juego. La Habana, Cuba: Vigot.

Mombaerts, E. (2000). Fútbol: del análisis del juego a la formación del jugador. Barcelona, España: INDE Publicaciones.

Morilla, M. y Gómez, M. A. (Coord.). (2009). Planificación del Trabajo Psicológico en Equipos de Fútbol. Sevilla, España: Difusión Gráfica.

Morilla, M. y Pérez, E. (Coord.) (2004). Entrenamiento de la Atención y Concentración. Ejercicios para su mejora ( $2^{\mathrm{a}}$ Ed.). Sevilla, España: Difusión Gráfica.

Morilla, M. y Rivera, S. (2014). La psicología en el entrenamiento integrado: diseño de actividades. En M. Roffé y S. Rivera. (Eds.). Entrenamiento Mental en el Fútbol Moderno: Herramientas Prácticas, (pp. 214-253). Barcelona, España: Fútbol de Libro.
Morilla, M., Rivera, S. y Tassi, J. M. (2017). Entrenamiento psicológico-integrado en la metodología del fútbol actual. Sevilla, España: Centro Excelentia.

Olmedilla, A. y Dominguez-Igual, J. (2016). Entrenamiento psicológico para la mejora de la atención y la autoconfianza en un futbolista. Revista de Psicología Aplicada al Deporte y al Ejercicio Físico, 1, E4. https://doi.org/10.5093/rpadef2016a4

Oña, A., Martínez, M., Moreno, F. y Ruiz, M. L. (1999). Control y aprendizaje motor. Madrid, España: Editorial Síntesis.

Parlebas, P. (1981). Contribution á un lexique commenté en sicience de l'action motrice. Paris, Francia: Insep.

Pascual, N., Alzamora, E. N., Martínez J. A. y Pérez, J. A. (2015). Análisis de los diferentes métodos de enseñanza utilizados en el fútbol base. Retos, 28, 94-97.

Pérez, V., Gutiérrez, M. T., García, A. y Gómez, J. (2005). Procesos psicológicos básicos: Un análisis funcional. Madrid, España: Pearson Educación.

Pulido, J. J., Sánchez, D., González, I., Amado, D., Montero, C., y García-Calvo, T. (2015). Adaptación y validación de un cuestionario para valorar la motivación en el contexto deportivo. Cuadernos de Psicología del Deporte, 15(3), 17-26.

Roffé, M. (1999). Psicología del jugador de fútbol. Buenos Aires, Argentina: Lugar Editorial S.A.

Roffé, M. y Rivera, S. (2014). Las diferentes inteligencias en la práctica del fútbol. En Autores (Eds.), Entrenamiento Mental en el Fútbol Moderno: Herramientas Prácticas (pp. 133-151). Barcelona, España: Futbol de Libro.

Sans, A. y Frattarola, C. (2009). Los fundamentos del fútbol: el entrenamiento basado en las situaciones de juego real. Mejora de la toma de decisiones para el desarrollo de un juego asociativo de posicionamiento. Programa AT-3. Pontevedra, España: MC Sports.

Seirul.lo, F. (2003). Sistemas Dinámicos y Rendimiento en Deportes de Equipo. 1st Meeting of Complex Systems and Sport. BarceIona, España: INEFC.

Tenenbaum, G. y Gershgoren, L. (2014). Individual and team decision making. En A. Papaioannou y D. Hackfort (Eds.), Fundamental concepts in sport and exercise psychology (pp. 458-477). Londres, Reino Unido: Taylor y Francis.

UEFA (2012). UEFA Euro 2012 Technical Report. Recuperado de: http://www.uefa.com/MultimediaFiles/Download/TechnicalReport/competitions/EURO/01/86/72/05/1867205_DOWNLOAD.pdf

UEFA (2016). UEFA Euro 2016 Technical Report. Recuperado de: http://www.uefa.com/MultimediaFiles/Download/TechnicalReport/competitions/EURO/02/40/26/69/2402669_DOWNLOAD.pdf

Vegas, G., Romero, R. y Pino, J. (2012). Metodología de la enseñanza en el fútbol basada en la implicación cognitiva. Sevilla: Sevilla, España: Wanceulen Editorial Deportiva. 


\section{Apéndice A: Ejemplo de tarea analítica.}

\section{OBJETIVOS}

Mejorar la comunicación y el apoyo social por medio de la circulación de balón.

\section{CONTENIDOS DE TRABAJO}

$\begin{array}{lll}\text { FísICOS: } & \text { TÉCNICOS: } & \text { TÁCTICOS: } \\ \text { Acondicionamiento previo } & \text { Control } & \text { Salida de balón } \\ \text { Dosificación: } & \text { Pase } & \\ 4 / 6^{\prime} & & \end{array}$

\section{PSICOLÓGICOS: acentuación componente psicológico:}

Motivación: reforzar las actitudes positivas.

Atención y concentración: sobre las indicaciones de los compañeros y la orientación corporal.

- Activación: aumentarla progresivamente.

- Cohesión grupal: ofrecer apoyo social con mensajes de aliento.

- Comunicación: expresar con claridad los mensajes verbales.

\section{DESCRIPCIÓN DE LA TAREA}

Se coloca un equipo completo o grupo numeroso para realizar pases y controles. A cada pase dado a un compañero, debe ir acompañado de una palabra de aliento e indicación de a quién pasar el balón. Puede realizarse buscando el orden en que debe circular al balón cuando este se saque jugado.

\section{CONSIGNAS}

Luego de realizar un pase, dar una voz de aliento o indicación al compañero que recibe.

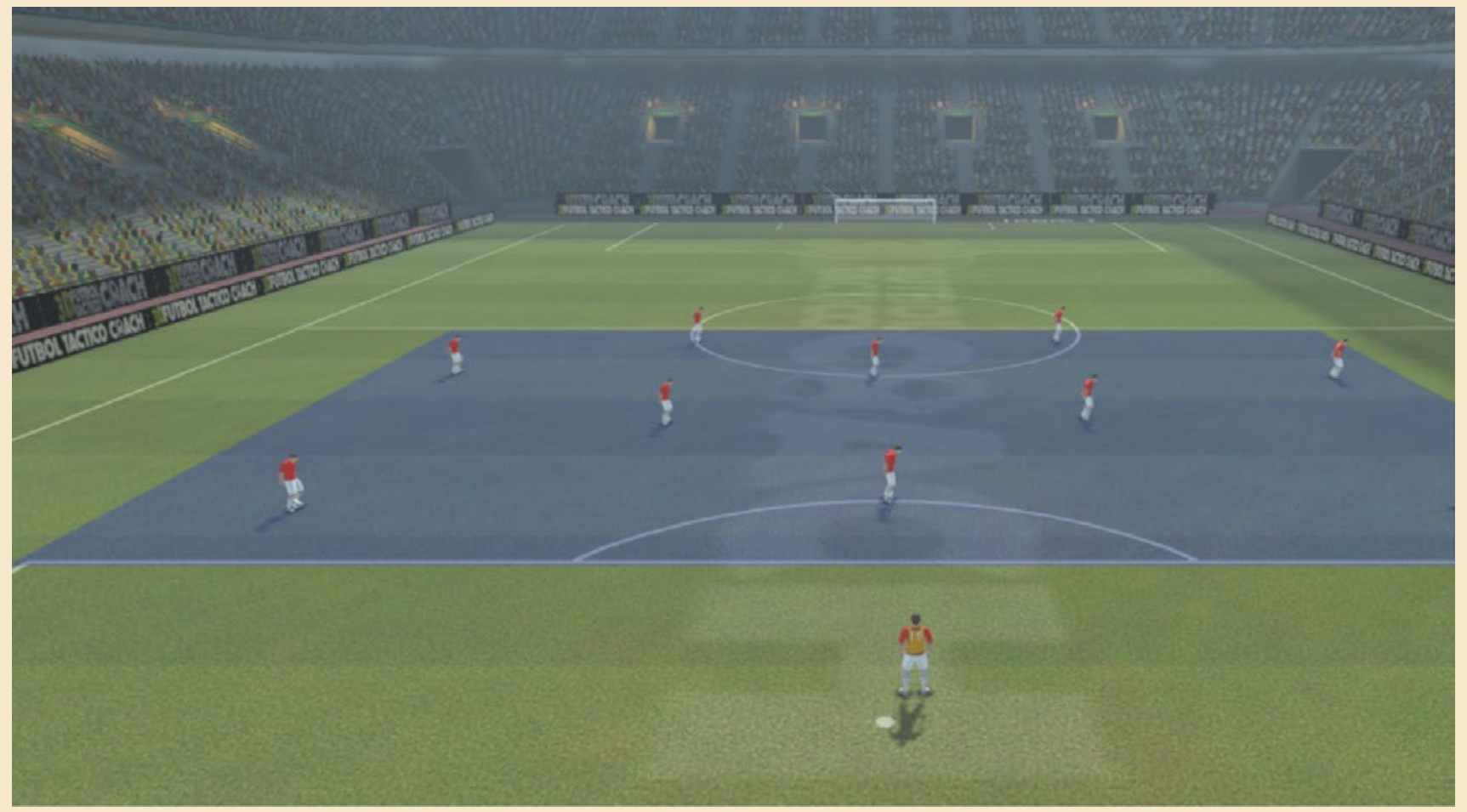




\section{Apéndice B: Ejemplo de tarea analítica integrada.}

\section{OBJETIVOS}

Ofensivos: conservar el balón y progresar utilizando la comunicación motriz y no verbal.

Defensivos: recuperar el balón sin hacer falta.

\section{CONTENIDOS DE TRABAJO}

FísıCos:

- Fuerza específica.

- Dosificación:

4 bloques de trabajo de $3 / 4^{\prime}$.

Macro pausa $1 / 2$
TÉCNICOS:

- Control

- Pase

- Desmarque

- Interceptación

\section{TÁCTICOS:}

- Conservación

Progresión

\section{PSICOLÓGICOS: acentuación componente psicológico:}

- Motivación: enfatizar la actitud competitiva y reforzar la correcta toma de decisiones.

- Atención y concentración: Ios atacantes focalizarán la atención en las transiciones, en los desmarques y los pases correctos; y los defensores en el esfuerzo por recuperar el balón.

- Control de la activación: atención a la agresividad defensiva sin hacer falta.

- Cohesión grupal: cuando se roten a los jugadores, reforzar el esfuerzo del defensor. Mejorar la comunicación motriz sin darles a los atacantes la posibilidad de hablar.

\section{DESCRIPCIÓN DE LA TAREA}

Inicia 2x1 dentro del cuadrado buscando progresar de lado a lado. Una vez que el balón logra pasar de un extremo al otro, salen otros dos jugadores en posesión y otro en defensa. En el caso de recuperar la defensa, inicia conservación hasta llegar a 5 toques con los jugadores ubicados sobre las esquinas. Gana quien logra sumar la mayor cantidad de progresiones y recuperaciones.

\section{CONSIGNAS}

3x1, 2 toques por jugador. Mínimo 3 pases entre compañeros por dentro. Al inicio los atacantes no pueden hablar. Rotar a los atacantes y defensores.

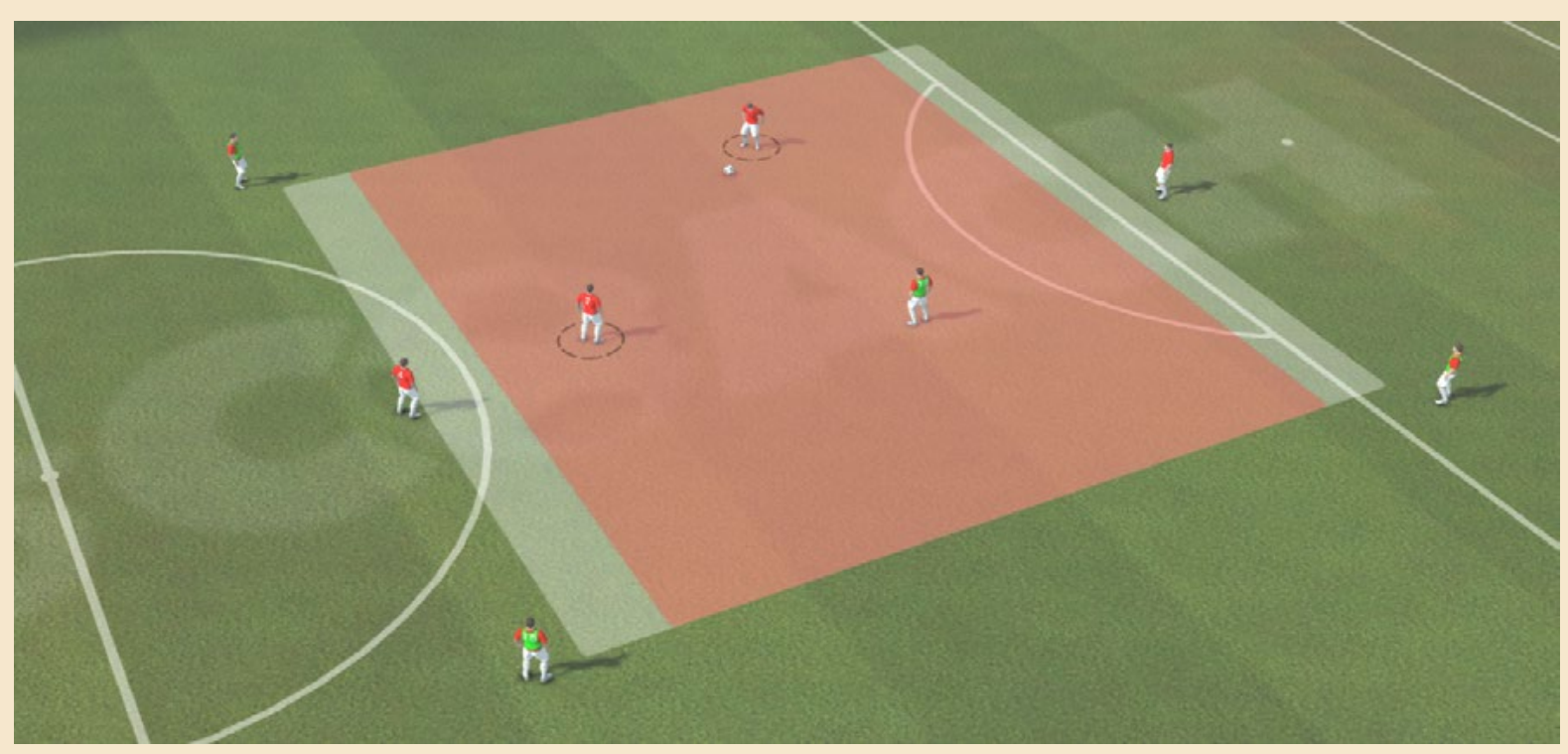




\section{Apéndice C: Ejemplo de tarea integrada global.}

\section{OBJETIVOS}

Ofensivos: conservar el balón enfatizando en la correcta orientación corporal y en la creación de líneas de pase, así como en la comunicación no verbal.

Defensivos: cerrar líneas de pase

\section{CONTENIDOS DE TRABAJO}

$\begin{array}{lll}\text { Físicos: } & \text { TÉCNICOS: } & \text { TÁCTICOS: } \\ \text { - Aptitud aeróbica y fuerza específica. } & \text { - Control } & \text { - Conservación } \\ \text { - Dosificación: } & \text { - Pase } & \text { - Creación de líneas de pase. } \\ 4 \text { bloques de trabajo de 3/4'. } & \text { - Finta } & \text { Apoyos } \\ \text { Macro pausa 1/2' } & \text { - Desmarque } & \end{array}$

\section{PSICOLÓGICOS: acentuación componente psicológico:}

Motivación: incidir en el correcto desarrollo de la tarea.

Atención y concentración: atender a los movimientos de los compañeros, oposición y ubicación del balón, buscando líneas de pase.

- Control de la activación: incidir en el ajuste de tensión cuando aumente la incertidumbre al haber 3 balones sin poder hablarse entre ellos.

Autoconfianza: reforzar la solución de situaciones más difíciles.

Cohesión grupal: incidir en el compromiso táctico al dar apoyos y en la comunicación no verbal (p.e. levantando el brazo o mostrándose en línea de pase)

\section{DESCRIPCIÓN DE LA TAREA}

En la zona marcada se ubican 12 jugadores con el mismo color de peto y otros 4 con blanco. Se utilizan dos balones al mismo tiempo (en algún momento pueden ser 3) tratando de pasar el balón a compañeros libres de jugadores con peto blanco que hacen sombra (no quitan el balón). Los jugadores de ambos equipos se mueven por todo el espacio siendo los blancos los que deben cambiar los jugadores a los que acercarse para obligar a los compañeros rojos a buscar otro compañero libre.

\section{CONSIGNAS}

1-2 toques de balón para pasar a compañero y pasar con la pierna más apropiada según la orientación corporal y ubicación del compañero; y movilidad para crear líneas de pase. Pedir que se hablen y por momentos eliminar este derecho, y aumentar el grado de oposición.

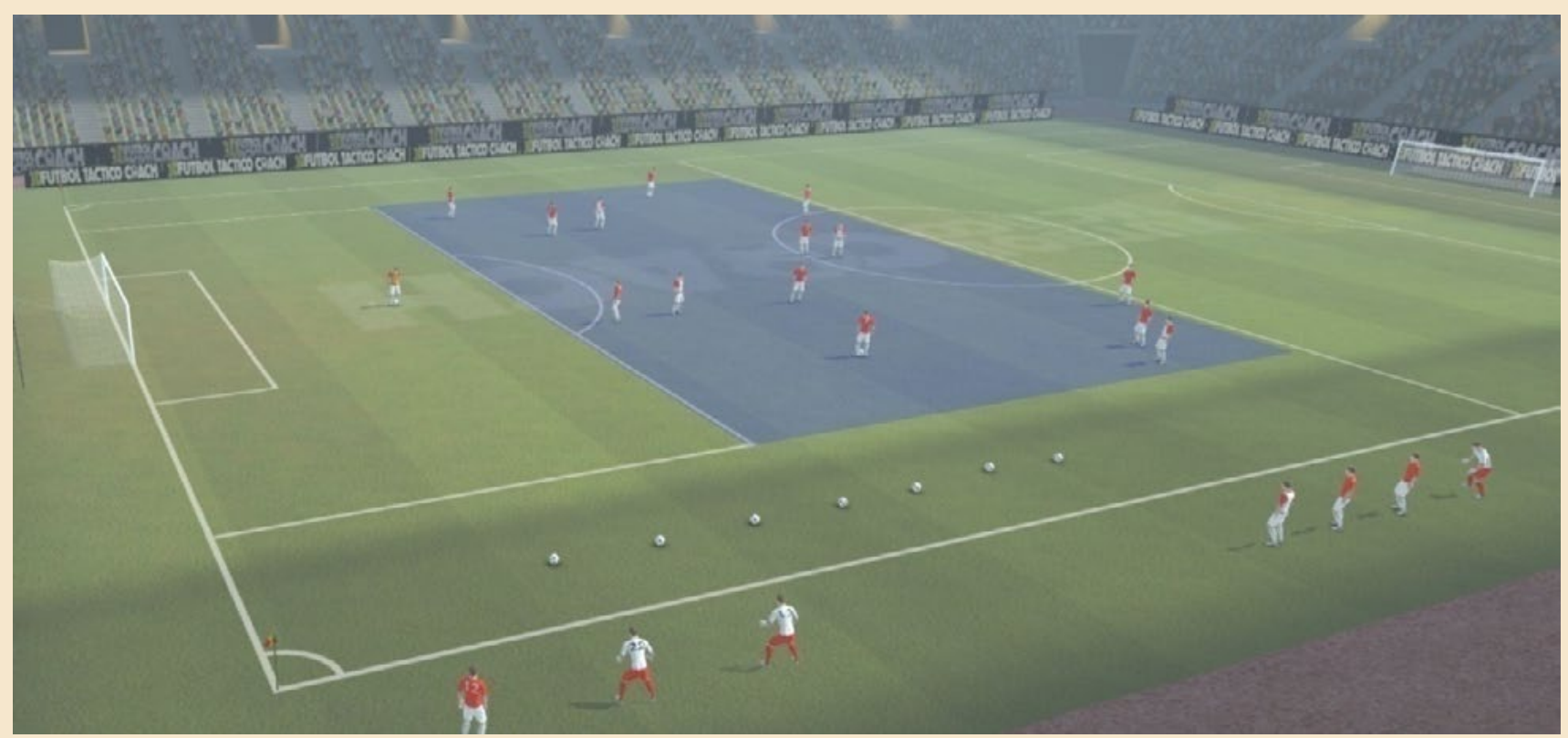

